\title{
Microbiota intestinal de indivíduos que sofreram acidente ocupacional com materiais biológicos e que realizaram profilaxia anti-retroviral
}

\author{
Intestinal microbiota of individuals who suffered occupational accidents \\ with biological materials and underwent antiretroviral prophylaxis
}

\author{
Micheli Evangelista de Souza ${ }^{1}$ e Paulo Câmara Marques Pereira ${ }^{1}$
}

\begin{abstract}
RESUMO
Avaliar a microbiota intestinal de indivíduos que sofreram acidente ocupacional com materiais biológicos e receberam anti-retrovirais foi o objetivo deste estudo. O grupo de estudo constou de 23 indivíduos com idade entre 18-45 anos, sendo 13 doadores de sangue e 10 que sofreram acidente ocupacional. Foram avaliados a microbiota intestinal, antropometria e exames laboratoriais pré, pós e 30 dias após o término da medicação. Zidovudina mais lamivudina foi utilizada em $70 \%$ dos indivíduos associado ao nelfinavir, $20 \%$ ao efavirenz e $10 \%$ ao ritonavir. As alterações nutricionais e dietéticas-laboratoriais e de microbiota intestinal foram analisadas em três momentos. M1: até dois dias do início da profilaxia; M2: no último dia da profilaxia e M3: 30 dias após o término da profilaxia. Náuseas, vômitos e diarréia estiveram presentes em $50 \%$ no segundo momento do estudo. Sobrepeso em $70 \%$, desnutrição e eutrofia em $10 \%$, dos indivíduos, não se modificaram durante o estudo. Transaminases, triglicérides, LDL-colesterol se elevaram no segundo momento e normalizaram 30 dias após término da medicação. Houve redução significativa dos Lactobacillus, Bifidobacterium e Bacteróides nos três momentos. Uso de anti-retrovirais provocou impacto significativo na microbiota intestinal dos indivíduos, sem recuperação em 30 dias.
\end{abstract}

Palavras-chaves: Microbiota intestinal. Anti-retroviral. Acidente ocupacional. Estado nutricional. HIV-1.

\begin{abstract}
The aim of this study was to evaluate the intestinal microbiota of individuals who had suffered occupational accidents and had received antiretrovirals. The study group consisted of 23 individuals between 18 and 45 years old, of whom 13 were blood donors and 10 had suffered occupational accidents. Intestinal microflora, anthropometry and laboratory tests were evaluated before, after and 30 days after discontinuation of the medication. Zidovudine plus lamivudine was used in association with nelfinavir for $70 \%$ of the individuals, with efavirenz for $20 \%$ and with ritonavir for 10\%. Nutritional, diet, laboratory and intestinal microbiota abnormalities were analyzed at three times: M1, not more than two days after starting prophylaxis; M2, on the last day of prophylaxis; and M3, 30 days after ending prophylaxis. Nausea, vomiting and diarrhea were present in 50\% at M2. Overweight in 70\%, malnutrition and eutrophy in 10\% of the individuals remained unchanged during the study. Transaminases, triglycerides and LDL-cholesterol because elevated at M2 and normalized 30 days after discontinuation of the medication. There were significant reductions in Lactobacillus, Bifidobacterium and Bacteroides at the three times. The use of antiretrovirals caused a significant impact on the individuals' intestinal microbiota, without recovery after 30 days.
\end{abstract}

Key-words: Intestinal microflora. Antiretroviral agents. Occupational accidents. Nutritional status. HIV-1.

0 trato gastrointestinal possui enorme variedade de bactérias aeróbias e anaeróbias que interagem entre si em complexo ecosistema ${ }^{14}$. 0 cólon apresenta flora extremamente rica, com aproximadamente 400-500 espécies de microorganismos e de bactérias anaeróbias, destacando-se em ordem decrescente: bacteróides, bifidobactérias, lactobacillus entre outros ${ }^{18}$.
Logo após o nascimento, o intestino, previamente estéril do lactente, começa a ser colonizado pelas bactérias da mãe e do meio ambiente. Durante os primeiros dias de vida, Escherichia coli, Clostridium e Estreptococos colonizam o trato gastrointestinal e com a amamentação surgem as bifidobactérias e os lactobacilos. Logo em seguida, aparecem os bacteróides, eubactérias e

\footnotetext{
1. Departamento de Doenças Tropicais, Faculdade de Medicina de Botucatu, Universidade Estadual Paulista, Botucatu, SP. Apoio financeiro: FAPESP - Fundação de Apoio a Pesquisa do Estado de São Paulo (Processo no 06/52649-7).

Endereço para correspondência: Prof. Paulo Câmara Marques Pereira. Dept ${ }^{0}$ de Doenças Tropicais/FMB/UNESP. Distrito de Rubião Junior s/ $\mathrm{n}^{0}$, 18618-000 Botucatu, SP. Tel: 55 14 3811-6212; Fax: $55143815-9898$

e-mail: ppereira@fmb.unesp.br

Recebido para publicação em: 12/03/2007

Aceito em: 17/10/2007
} 
peptococos ${ }^{4}$. 0 recém nascido desenvolve coleção heterogênea de bactérias no trato gastrointestinal, sob a influência de fatores relacionados ao hospedeiro, as bactérias e outros fatores externos, como o uso de medicamentos, alimentação, estado nutricional, e condições de higiene/contaminação ambiental ${ }^{17}$. A microbiota gastrointestinal permanece relativamente estável durante a vida do indivíduo $0^{313}$. As principais funções atribuídas à microbiota intestinal são: antibacteriana, imunomoduladora e metabólico/ nutricional ${ }^{17}$.

O uso de antimicrobianos, podem causar alterações no padrão de colonização intestinal. 0 principal efeito observado inclui a supressão relevante das bactérias anaeróbias, com exceção do Clostridium sp que permanece em níveis detectáveis. A influência dessas drogas na microbiota intestinal é transitória, mas pode persistir por algum período após o término do uso $0^{911}$. A redução da população bacteriana intestinal pode ocasionar alterações imunológicas entre outras, as quais dependem muitas vezes da concentração da droga no lúmen intestinal e de seu espectro de ação $0^{11}{ }^{15}$. É difícil avaliar a participação da doença como fator associado nessas alterações ${ }^{8}$.

Monreal cols ${ }^{14}$ observaram em estudo recente que pacientes com infecção de vias aéreas em uso de amoxicilina revelaram redução significativa da quantidade de Lactobacillus $\boldsymbol{e}$ Bibidobacterium. Também, foi observado que após 30 dias do término do tratamento esses microorganismos retornaram ao normal

Embora existam poucos trabalhos sobre a possível interação entre a infecção do HIV/aids e o uso de anti-retrovirais na microbiota intestinal, não há relatos da ação isolada do uso de drogas anti-retrovirais na ausência de infecção ou doença, como em indivíduos que sofreram exposições ocupacionais com materiais biológicos.

As exposições ocupacionais com materiais biológicos e os ferimentos com agulhas e material pérfuro-cortante, em geral, são considerados extremamente perigosos por serem potencialmente capazes de transmitir mais de 20 tipos de patógenos diferentes ${ }^{2}$, sendo o vírus da imunodeficiência humana (HIV), da hepatite $\mathrm{B}$ e da hepatite $\mathrm{C}$ os agentes infecciosos mais comumentes envolvidos ${ }^{6}$.

0 risco de aquisição do HIV pós-exposição ocupacional percutânea com sangue contaminado é de aproximadamente 0,3\% e de $0.09 \%$ envolvendo exposições de mucosa. 0 risco após exposição envolvendo pele não íntegra, não é precisamente quantificado, estimando-se que ele seja inferior ao risco das exposições em mucosas ${ }^{12}$. A indicação da quimioprofilaxia deverá ser iniciada o mais rápido possível, dentro de uma a duas horas após o acidente e a duração da quimioprofilaxia é de quatro semanas. O uso combinado de zidovudina (AZT) com lamivudina (3TC) é recomendado na maioria das situações com indicação de uso de quimioprofilaxia. 0 uso do indinavir ou nelfinavir deve ser reservado para acidentes graves e situações em que haja possibilidade de resistência viral do paciente-fonte ${ }^{14}$.

Há necessidade de se conhecer melhor as possíveis interações dessas drogas na microbiota intestinal e assim contribuir para o melhor seguimento desses indivíduos.

\section{MATERIAL E MÉTODOS}

Estudo prospectivo com 23 indivíduos distribuídos em dois grupos: Grupo Controle (GC): 13 indivíduos doadores de sangue; Grupo 1 (G1): 10 indivíduos que sofreram acidente ocupacional com materiais biológicos e realizaram profilaxia anti-retroviral durante 28 dias atendidos no Hospital das Clínicas da Faculdade de Medicina de Botucatu/Universidade Estadual Paulista-UNESP. Foram incluídos todos os indivíduos que sofreram acidente ocupacional com materiais biológicos e realizaram profilaxia anti-retroviral. Foram excluídos menores de 18 anos, gestantes e lactantes, indivíduos em uso prévio de antimicrobianos nos últimos 30 dias, e os que interromperam a profilaxia durante o estudo.

Avaliações nutricionais e dietéticas, laboratoriais e da microbiota intestinal ocorreram em três momentos. M1: até dois dias do início da profilaxia, M2: no último dia da profilaxia e M3: 30 dias após o término na medicação nos indivíduos do G1. A avaliação nutricional foi obtida através de medidas antropométricas considerando o índice de massa corpórea (IMC) para a classificação do estado nutricional ${ }^{19}$ e análise alimentar de macronutrientes carboidratos, proteínas, lipídios e fibras obtidos através do cálculo com o software Diet Pró da Faculdade Federal de Viçosa. A avaliação laboratorial dos níveis séricos de proteínas totais e frações, uréia e creatinina, glicemia, lipidograma, enzimas hepáticas e hemograma também foram realizados.

A avaliação da microbiota intestinal foi realizada pelo método adaptado por Sutter cols ${ }^{16}$. No momento da anamnese, foram entregues aos indivíduos frascos de coleta de fezes estérel e um frasco contendo $9 \mathrm{ml}$ de meio de transporte, para a avaliação nos três momentos do estudo. Após a coleta da amostra, foi pesado um grama de fezes e realizada a diluição em $9 \mathrm{ml}$ de meio de Stuart transporte médium de $10^{2}$ a $10^{8}$. Após a homogeinização foi realizado o plaqueamento em meios específicos de Kanamicyn vancomicyn blood agar, Bifidobacterium médium, Lactobacillus seletive e acondicionado em jarra de anaerobiose Gaspak com gerador de anaerobiose Anaerobac (Probac) por 48 horas em incubadora a $37^{\circ} \mathrm{C}$. Após este período foi realizada a contagem das unidades formadoras de colônias (UFC) em contador de colônias. A concentração de microorganismos foi calculada em $\log _{10}$ UFC/g de fezes.

Foi realizado teste pareado nos três momentos do estudo para o grupo 1. Para contagem de Bacteróides, Bifidobacterium e Lactobacillus, foram calculados média e desvio padrão com transformação logarítmica dos dados e média em escala original. Na comparação com o grupo GC foram utlizados teste $t$ para duas amostras independentes para o cálculo das estatísticas t e p não pareados. Foram considerados significativos quando $\mathrm{p} \geq 0,05$; para a análise foi utilizado o programa SSPS versão 14.0.

0 presente estudo recebeu parecer favorável pelo Comitê de Ética em Pesquisa da Faculdade de Medicina de Botucatu da Universidade Estadual Paulista. 


\section{RESULTADOS}

Dos 23 indivíduos estudados, houve predomínio do sexo feminino em ambos os grupos, sendo 70\% no grupo de indivíduos que receberam anti-retrovirais (G1) e 53\% no grupo controle (GC). A faixa etária predominante ficou entre 18-40 anos de idades em ambos os grupos, 54\% no GC e 60\% no GC.

Avaliação do estado nutricional quanto a composição corporal, índice de massa corporal, porcentagem de gordura e circunferência muscular do braço não mostraram diferenças entres os grupos nos diferentes momentos analisados, porém encontrou-se predomínio de sobrepesos no G1 e eutrofia no GC.

A avaliação dietética realizada pela análise do recordatório alimentar no $\mathrm{G} 1$ revelou ingestão reduzida de carboidratos e fibras e ingestão excessiva de lipídios e proteínas.

Não houve diferença estatística na comparação dos níveis séricos de uréia, creatinina, colesterol total e HDL colesterol, proteínas totais e frações, glicemia, hematócrito e hemoglobina entres os momentos avaliados, estando todos com valores normais. As enzimas hepáticas AST, ALT, Gama-GT bem como LDL colesterol e triglicérides apresentaram diferença estatística entre os momentos avaliados conforme descrito na Tabela 1 .

A ocorrência de acidentes percutâneos foi de $80 \%$, seguido de mucosa 10\% e cutâneo 10\%. A combinação de AZT+ 3TC fez parte de todos os esquemas profiláticos. Observou-se que os efeitos adversos provocados pela quimioprofilaxia foram náuseas, vômito e diarréia em 50\% dos indivíduos no M2.

A avaliação da microbiota intestinal bem como a análise estatística encontram-se descritas na Tabela 2 e ilustradas na Figura 1. A concentração de Lactobacillus, Bifidobacterium e Bacteroides foi menor do que no grupo controle em todos os momentos.

Tabela 1 - Caracterização dos exames laboratoriais em média e desvio padrão dos 10 indivíduos do G1 entre os três momentos.

\begin{tabular}{lccc}
\hline $\begin{array}{l}\text { Momentos } \\
\text { Níveis séricos }\end{array}$ & $\begin{array}{c}\mathrm{M} 1 \\
(\mathrm{x} \pm \mathrm{s})\end{array}$ & $\begin{array}{c}\mathrm{M} 2 \\
(\mathrm{x} \pm \mathrm{s})\end{array}$ & $\begin{array}{c}\mathrm{M} 3 \\
(\mathrm{x} \pm \mathrm{s})\end{array}$ \\
\hline LDL colesterol (mg/dL) & $98.55 \pm 20,59$ & $130,11 \pm 47,65$ & $112,82 \pm 34,01$ \\
Triglicérides (mg/dL) & $143,48 \pm 64,48$ & $146,22 \pm 103,69$ & $135,67 \pm 58.83$ \\
AST $(\mathrm{u} / \mathrm{L})$ & $34,10 \pm 7.73$ & $43,89 \pm 21.84$ & $26,60 \pm 9,02$ \\
ALT $(\mathrm{u} / \mathrm{L})$ & $23,10 \pm 4,43$ & $25,56 \pm 25,56$ & $21,40 \pm 5,17$ \\
Gama-GT $(\mathrm{u} / \mathrm{L})$ & $37,00 \pm 27,37$ & $66,00 \pm 48,75$ & $29,70 \pm 18,25$ \\
\hline Hipótese & Estatística & Comentário \\
\hline LDL-colesterol: $\mathrm{M} 1=\mathrm{M} 2 ; \mathrm{M} 2=\mathrm{M} 3 ; \mathrm{M} 1=\mathrm{M} 3$ & $\mathrm{p}<0,05$ & $\mathrm{M} 1<\mathrm{M} 2$ \\
Triglicérides: $\mathrm{M} 1=\mathrm{M} 2 ; \mathrm{M} 2=\mathrm{M} 3 ; \mathrm{M} 1=\mathrm{M} 3$ & $\mathrm{p}<0,05$ & $\mathrm{M} 2>\mathrm{M} 3$ \\
AST: M1=M2; M2=M3; M1=M3 & $\mathrm{p}<0,05$ & $\mathrm{M} 2>\mathrm{M} 3$ \\
& & $\mathrm{p}<0,05$ & $\mathrm{M} 2>\mathrm{M} 3$ \\
ALT: M1=M2; M2=M3; M1=M3 & $\mathrm{p}<0,05$ & $\mathrm{M} 2>\mathrm{M} 3$ \\
\hline Gama-GT : M1=M2; M2=M3; M1=M3 & &
\end{tabular}

( $\mathrm{x} \pm \mathrm{s})$ média e desvio padrão.

GC: grupo controle-indivíduos doadores de sangue, G1: indivíduos que sofreram acidente ocupacional com materiais biológicos que realizaram de profilaxia antiretroviral.

M1: até dois dias após o inicio da profilaxia, M2: no último dia da profilaxia, M3: 30 dias após o término da medicação.

LDL: lipoproteinas de baixa densidade, AST: aspartato aminotransferase, ALT: alanina amino transferase, Gama-GT: gama-glutamiltransferase.
Tabela 2 - Média e desvio padrão $(x \pm s)$ da contagem logarítmica de Lactobacillus Bifidobactérium e Bacteróides dos grupos GC versus G1 nos três momentos.

\begin{tabular}{|c|c|c|c|c|}
\hline Momentos & M1 & M1 & M2 & M3 \\
\hline \multirow[t]{2}{*}{ Grupos } & GC & G1 & G1 & G1 \\
\hline & $(\mathrm{x} \pm \mathrm{s})$ & $(\mathrm{x} \pm \mathrm{s})$ & $(\mathrm{x} \pm \mathrm{s})$ & $(\mathrm{x} \pm \mathrm{s})$ \\
\hline Lactobacillus $(\log / \mathrm{g})$ & $9,57 \pm 0,85$ & $7,6 \pm 0,371$ & $6,96 \pm 0,89$ & $6,60 \pm 43$ \\
\hline Bifidobacterium $(\log / g)$ & $10,55 \pm 0,93$ & $9,0 \pm 0,809$ & $8,37 \pm 1,39$ & $8,14 \pm 1,39$ \\
\hline Bacteroides (log/g) & $11,68 \pm 0,459$ & $9,33 \pm 0,68$ & $8,06 \pm 2,93$ & $8,96 \pm 0,66$ \\
\hline \multicolumn{3}{|l|}{ Hipótese } & Estatística & Comentário \\
\hline \multicolumn{5}{|c|}{ Interação entre os grupos $\quad$ (GC e G1) } \\
\hline \multirow[t]{3}{*}{ entre os momentos. } & \multicolumn{2}{|c|}{$\mathrm{GC}>\mathrm{G} 1(\mathrm{M} 1)$} & $\mathrm{p}<0,05$ & $\mathrm{GC}>\mathrm{G} 1(\mathrm{M} 1)$ \\
\hline & \multicolumn{2}{|c|}{$\mathrm{GC}>\mathrm{G} 1(\mathrm{M} 2)$} & $\mathrm{p}<0,05$ & $\mathrm{GC}>\mathrm{G} 1(\mathrm{M} 2)$ \\
\hline & \multicolumn{2}{|c|}{$\mathrm{GC}>\mathrm{G} 1(\mathrm{M} 3)$} & $\mathrm{p}<0,05$ & $\mathrm{GC}>\mathrm{G} 1(\mathrm{M} 3)$ \\
\hline \multicolumn{4}{|c|}{ Diferença do G1 entre os momentos (M1, M2 e M3) } & $\mathrm{M} 1>\mathrm{M} 2$ \\
\hline \multicolumn{3}{|l|}{ Lactobacillus } & $\mathrm{p}<0,05$ & M1 $>$ M3 \\
\hline \multicolumn{5}{|c|}{ Diferença do G1 entre os momentos (M1, M2 e M3) } \\
\hline \multicolumn{3}{|l|}{ Bifidobacteium } & $\mathrm{p}<0,05$ & $\mathrm{M} 1>\mathrm{M} 3$ \\
\hline \multicolumn{4}{|c|}{ Diferença do G1 entre os momentos (M1, M2 M3) } & $\mathrm{M} 1=\mathrm{M} 2$ \\
\hline \multirow[t]{2}{*}{ Bacteroides } & & & $p>0,05$ & $\mathrm{M} 2=\mathrm{M} 3$ \\
\hline & & & & $\mathrm{M} 3=\mathrm{M} 1$ \\
\hline
\end{tabular}

( $\mathrm{x} \pm \mathrm{s})$ média e desvio padrão.

GC: Grupo controle-indivíduos doadores de sangue, G1: indivíduos que sofreram acidente ocupacional com materiais biológicos que realizaram de profilaxia antiretroviral.

M1: até dois dias após o início da profilaxia, M2: no último dia da profilaxia, M3: 30 dias após o término da medicação.

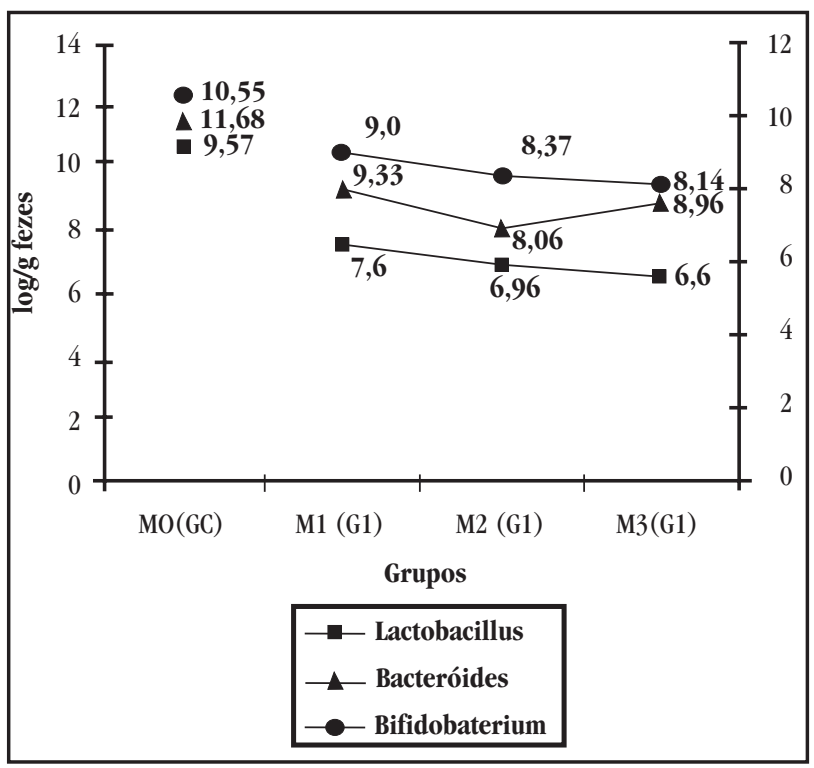

Figura 1-Média logarítmica por g/fezes de Lactobacillus, Bifidobacterum e Bacteroides dos indivíduos do G1 versus GC entre os três momentos considerando nível de significância $p<0,05$.

\section{DISCUSSÃO}

0 trato gastrointestinal é um sistema altamente complexo que tem despertado interesse de muitos pesquisadores principalmente associado a presença de infecção ${ }^{13}{ }^{14}{ }^{18}$. Segundo Brandt cols, vários fatores podem influenciar a colonização da microbiota intestinal tais como genética, idade, sistema imune, alimentação, estado nutricional e uso de antimicrobianos ${ }^{5}$. 
No presente estudo, as variáveis idades, sexo, alimentação e o estado nutricional, não interferiram significativamente sobre a microbiota intestinal dos indivíduos. A dieta não demonstrou modificação com a utilização dos anti-retrovirais, porém observouse ingestão excessiva de lipídios e proteínas e baixa ingestão de fibras, que não se modificaram nos diferentes momentos o que não deve ter interferindo nos resultados obtidos.

0 grupo de indivíduos que recebeu anti-retrovirais, apresentou percentual de gordura acima do intervalo de referência e sobrepeso sem alteração durante 0 estudo, consequiência associada a alta ingestão alimentar de gorduras e sedentarismo.

0 uso do AZT + 3TC esteve associado a todos os esquemas utilizados, segundo recomendação do Ministério da Saúde por ter eficácia comprovada e boa tolerância digestiva ${ }^{12}$.

Os efeitos adversos encontrados no estudo pelo uso da quimioprofilaxia no trato gastrointestinal não diferiram dos relatos da literatura ${ }^{12}$.

As elevações nos níveis séricos das transaminases, AST, ALT e Gama-GT bem como nos triglicérides e LDL-colesterol, que ocorreram no M2, se normalizaram trinta dias após o término da quimioprofilaxia. Esses dados concordam com os relatos da literatura ${ }^{12} 18$.

No presente estudo, a redução das bactérias anaeróbias Bacteroides, Bifidobacterium e Lactobacillus nos indivíduos em uso de anti-retrovirais, nos três momentos, foi importante durante todo o seguimento.

Os lactobacilos e as bifidobactérias, são encontrados em grandes quantidades na microbiota intestinal anaeróbia. Esses microorganismos contribuem para a manutenção da resistência à colonização, pela produção de ácidos acéticos e lácticos, que diminuem o pH intestinal, impedindo o crescimento excessivo de outros microrganismos, potencialmente patogênicos, que têm seu crescimento limitado pela acidez intestinal'.

Os Lactobacillus e Bifidobacterium, mantiveram-se reduzidos após 30 dias do término da profilaxia, revelando a importância de se estudar melhor essas variáveis para possíveis intervenções. Cabe salientar que outros estudos com antibióticos e infecções que não HIV/aids tem mostrado que ocorre normalização da microbiota até 30 dias após o tratamento o que não foi observado neste trabalho.

Os anti-retrovirais apresentaram impacto importante na microbiota intestinal e já no primeiro momento, pode-se identificar alterações na mesma que se intensificaram durante a profilaxia e não se normalizaram após o término do uso dos anti-retrovirais.

Sabendo-se da importância da microbiota intestinal no desempenho de atividades antibacterianas, imunomoduladoras e metabólico-nutricionais, novos estudos devem ser realizados e associados à intervenção nutricional, no sentido se de aprofundar os conhecimentos da interação entre droga e microbiota intestinal, e assim propor medidas para reduzir esse desequilíbrio, o qual pode ser extremamente prejudicial aos indivíduos.

\section{AGRADECIMENTOS}

Agradecemos à Fundação de Apoio a Pesquisa do Estado de São Paulo, pelo apoio financeiro para a realização deste trabalho.

\section{REFERÊNCIAS}

1. Bartllet JG. Antibiotic-associated diarrhea. Clinical Infection Diseases 15: 157-164, 1992.

2. Beltrami EM, Williams LT, Shapiro N, Chamberland ME. Risck and management of blood-borne infections in health care workers. Clinical Microbiology Reviews 23: 345-348, 2000.

3. Berg RD. The indigenous gastrointestinal microflora. Trends Microbiology 4: 430-435, 1996.

4. Bourlioux P, Koletze B, Guarner F, Braesco V. The intestine and its microflora are patners for the protection of the host: repart on the Danone Symposium "The Intelligent Intestine”, 2002. American Journal Clinical Nutrition 73: 675-683, 2006.

5. Brandt KG, Sampaio Magda MSC, Miuki CJ. Importância da microflora intestinal. Pediatria 28:117-127, 2006.

6. Cardo DM, Culver DH, Ciesielki, CA. A case Control study of HIV seroconversion in health care workers after percutâneos exposure. New England Journal of Medicine 337: 1485-1490, 1997.

7. Cummings JH, Macfarlane GT. The control and consequences of bacterial fermentation in the human colon. Journal Applied Bacteriology 70: 443459,1991 .

8. Gooch WM, Blair E, Puopolo A. Effectiveness of five days of terapy with cefuroxime axetil suspension of treatment of acute otitis media. Pediatric Infectious Disease Journal 15: 157-154, 1996.

9. Gorbach SL, Sparknebel G, Weinstein L, Plaut AG, Nahas L, Levitan R. Studies of intestinal microflora. VIII. Effect of Lincomycin on the microbial population of the human intestine. Journal of Infectious Diseases 120: 298-304, 1969.

10. Lidbeck A, Nord CE. Lactolacilli and the normal human anaerobic microflora. Clinical Infectious Diseases 16: 181-187, 1993.

11. Mackowiak PA. The normal microbial flora. New England Journal of Medicine 307: 83-93, 1982.

12. Ministério da Saúde. Recomendações para atendimento e acompanhamento de exposições ocupacional a material biológico: HIV e Hepatites B e C. Brasília, 2006

13. Mitsuoka T. Intestinal flora and aging. Revista de Nutrição 50: 438-446, 1992.

14. Monreal MTFD, Pereira PCM, Lopes CAM. Intestinal microbiota of patients with bacterial infection of the respiratory tract with Amoxicillin. Brazilian Journal Infectious Diseases 9: 292-300, 2005.

15. Nondervall B, Hallberg D, Larsson L, Nord CE. The effect of clindamycin on the intestinal flora in patients with enteric hiperoxaluria. Scandinavian Journal of Gastroenterology 18: 177-181, 1983.

16. Sutter VL, Citron DM, Finegold SM, Brichnell KS. Wadsworth anaerobic bacteriology manual. Departament of Continuing Education in Health Sciences, University Extension, and the School of Medicine, University of California: CV Mosby; Company, California, 1972.

17. Toivanen P, Vaahtovuo J, Eerola E. Iinfluence of major histocompatibility complex on bacterial composition of fecal flora. Infection and Immunity 69: 2372-2377, 2001.

18. Trindade EBSM. Avaliação da flora bacteriana intestinal e do estado nutricional de indivíduos infectados pelo HIV-1, suplementados com fibra solúvel e probiótico. Tese de Doutorado, Botucatu. Faculdade de Medicina, Universidade Estadual Paulista, 2004.

19. Word Health Organization. Report of a WHO Expert Commite Physical status the use and interpretation of anthropometry. Geneva, 1995. 